\title{
Session orale pédiatrique (1)
}

\section{Paediatric oral session (1)}

(C) SRLF et Springer-Verlag France 2013
Conclusion: In this study, the children and the ventilator were poorly synchronized. Asynchrony was not associated with MV duration. Unexpectedly, the ventilatory drive was frequently blunted. Strategies to improve synchronisation should be evaluated and future studies should investigate the clinical impact of respiratory inactivity in children.

\section{SO020}

Feasibility of neurally adjusted ventilatory assist (NAVA) during pediatric Non-Invasive Ventilation

L. Ducharme-Crevier ${ }^{1}$, J. Beck ${ }^{2}$, P. Jouvet ${ }^{1}$, G. Emeriaud ${ }^{1}$

${ }^{1}$ Service de soins intensifs pédiatriques, CHU Sainte-Justine,

Montréal, Canada

${ }^{2}$ Li Ka Shing Knowledge Institute, St Michael'S Hospital, University of Toronto, Toronto, Canada

Introduction: Non-invasive ventilation (NIV) permits to support the ventilation while limiting complications related to intubation. However, NIV fails in up to half children with acute respiratory failure. The ventilator's inability to detect patient effort is one major cause of NIV failure. Neurally adjusted ventilatory assist (NAVA), a mode of ventilation controlled by the patient's neural respiratory drive, improves patient ventilator interaction during invasive ventilation. We hypothesized that non-invasive NAVA (NIV-NAVA) is feasible in pediatric patients and permits to achieve a good patient-ventilator synchrony.

Patients and methods: Prospective crossover study. Patients 1 month to 18 years old, requiring NIV in the pediatric ICU were eligible. After parental consent, patients were ventilated successively in conventional NIV (30 minutes, settings as prescribed by attending physician), with NIV-NAVA (60 min.) and again with conventional NIV (30 min). Electrical activity of the diaphragm (EAdi) and airway pressure (Pvent) were simultaneously recorded during these periods using validated recording methods. EAdi and Pvent plots were analyzed semi-automatically to identify the timings of patient efforts and ventilatory support. The asynchrony variables (including inspiratory trigger delay, cycling-off delay, auto-triggered breath and wasted effort) were compared using the non-parametric Friedman test.

Results: 12 patients, mean \pm SD age of $60 \pm 45$ months (range: 1 month-5 years) were included. NIV-NAVA was possible and well tolerated in all patients. During conventional NIV, 4 patients were on continuous positive pressure (CPAP, $7.2 \pm 0.8 \mathrm{cmH}_{2} \mathrm{O}$ ), and 8 patients had a bi-level support (peep $5.2 \pm 2.2$, pressure support $9.9 \pm 3.5 \mathrm{cmH}_{2} \mathrm{O}$ ). During NAVA ventilation, the peep was $6.1 \pm 1.6$ and NAVA level was $0.5 \pm 0.4 \mathrm{cmH}_{2} \mathrm{O} / \mathrm{mcV}$ ). As illustrated in the Figure, all synchrony parameters significantly improved during NAVA (all $\mathrm{p}<0.05$ ).

No significant change of inspiratory EAdi was observed in the entire group, but EAdi decreased during NAVA in patients with high baseline EAdi $(>10 \mathrm{mcV})$, whereas it increased in patients with low baseline EAdi $(<5 \mathrm{mcV})(\mathrm{p}<0.05$ for interaction between EAdi change 


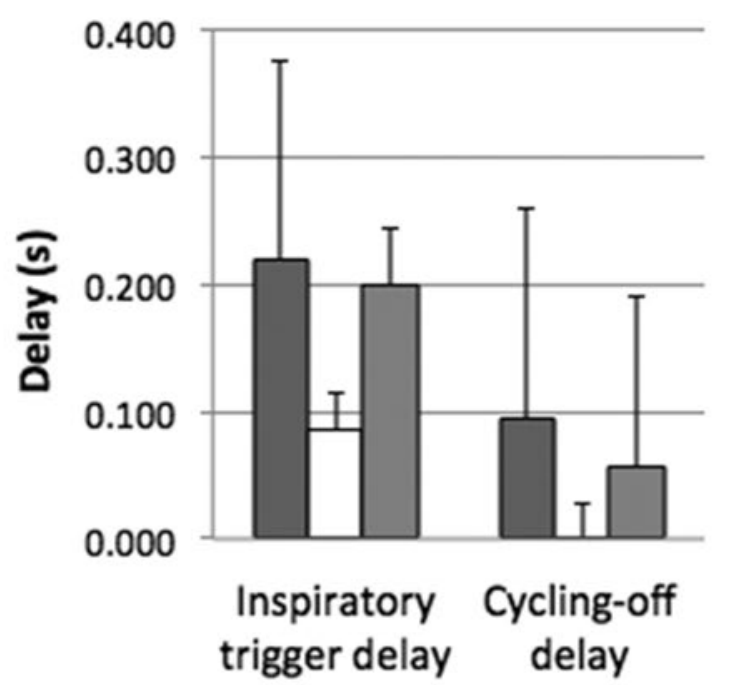

and baseline EAdi). One adolescent asked to stop the study because of anxiety related to the mask. This patient was trained with conventional NIV at home with a nasal mask with leaks (prior to scoliosis surgery), and the mask used in this study was a naso-buccal leak-free mask.

Discussion: The order of NIV modes was not randomized but the second period of conventional NIV suggest that a temporal effect is unlikely. The study size and the short-term evaluation do not permit to evaluate the impact of NAVA on major clinical outcome data (NIV success rate or duration).

Conclusion: NIV NAVA seems feasible in children with acute respiratory failure and allows good patient-ventilator synchronization, which is a significant improvement in pediatric NIV. Larger controlled studies are warranted to evaluate the impact of these findings on NIV success rate and duration.

\section{SO021}

\section{Ventilation non invasive dans la bronchiolite sévère du nourrisson en échec de CPAP:}

NAVA versus pression assistée contrôlée

F. Baudin ${ }^{1}$, R. Pouyau ${ }^{1}$, F. Cour-Andlauer ${ }^{1}$, J. Berthiller ${ }^{2}$,

D. Robert ${ }^{3}$, E. Javouhey ${ }^{1}$

${ }^{1}$ Réanimation pédiatrique, CHU de Lyon-Hfme, Bron, France

${ }^{2}$ Recherche clinique, CHU de Lyon-Hfme, Bron, France

${ }^{3}$ Réanimation, CHU de Lyon-Hôpital Édouard-Herriot, Lyon, France

Introduction : La Continuous Positive Airway Pressure (CPAP) est la technique d'assistance respiratoire de référence pour la prise en charge des bronchiolites aigues chez le nourrisson. En cas d'échec, le recours à la ventilation non invasive (VNI) est utilisé par certaines équipes [1] mais la synchronisation patient-respirateur reste un problème majeur qui semble amélioré par la Neurally Adjusted Ventilatory Assist (NAVA) chez l'adulte et chez l'enfant [2]. L'objectif de cette étude est d'évaluer la faisabilité de la NAVA en VNI et son impact sur la synchronisation patient-respirateur chez le nourrisson atteint de bronchiolite.

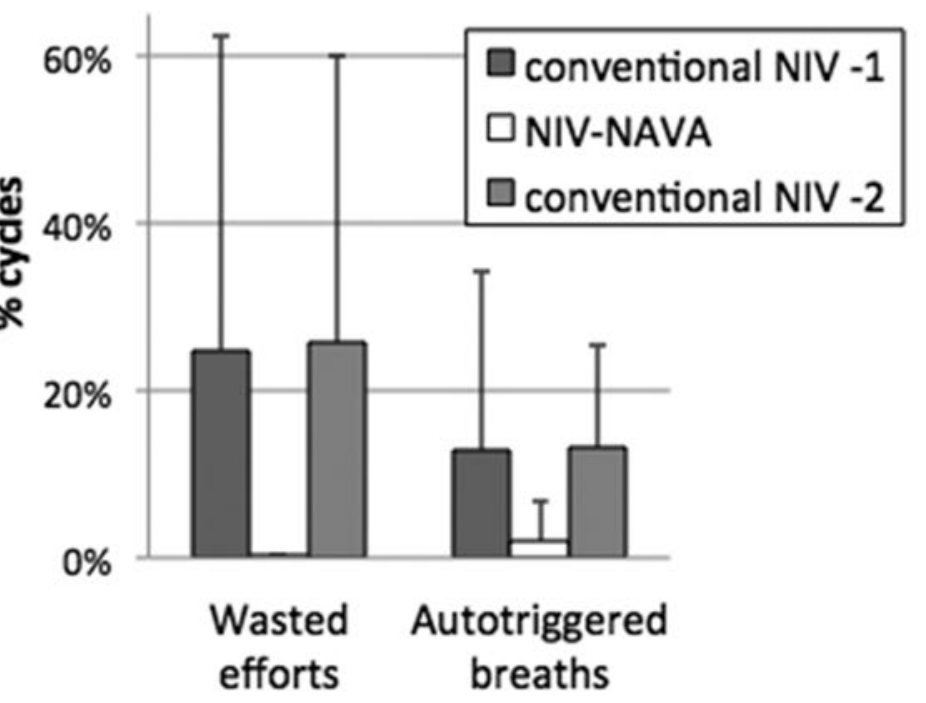

Patients et méthodes : Étude prospective menée entre décembre 2012 et mai 2013. Inclusion des enfants de moins de 6 mois VRS positif en échec de prise en charge en CPAP. Deux périodes successives d'observation de $2 \mathrm{~h}$ sont effectuées en mode pression assistée contrôlée PAC puis en mode NAVA. La fréquence respiratoire (FR), les principales asynchronies (auto-déclenchement, double déclenchement, effort inefficace), le niveau Eadi, le délai de déclenchement du respirateur étaient analysés durant 1 min cycle à cycle à partir de l'analyse des courbes de pression et d'activité électrique diaphragmatique (Eadi). Les données cliniques et biologiques étaient recueillies au début de chaque période. Les résultats sont exprimés en moyenne (écart-type). Les données ont été comparées au moyen du test de Student pour données appariées après vérification de la normalité.

Résultats : 11 enfants âgés de 35,2 \pm 23 jours et pesant $3,73 \pm 0,7 \mathrm{~kg}$ ont été inclus. La durée moyenne de ventilation en CPAP avant admission était de 12,36 $\pm 10,1 \mathrm{~h}$. Les données cliniques (Fréquence cardiaque, $\mathrm{SpO} 2, \mathrm{~m}$-WCAS) n'étaient pas différentes entre les 2 périodes. Les principaux résultats sont présentés dans le Tableau 1.

Discussion : L'index d'asynchronie est significativement diminué en mode NAVA de même que le délai de réponse. Les limites principales sont le nombre limité de patients inclus et l'absence de randomisation des séquences d'observation.

Conclusion : La NAVA est un mode utilisable pour la VNI chez les enfants atteints de bronchiolite en échec de CPAP et permet d'améliorer significativement la synchronisation inspiratoire et le délai de réponse du respirateur. D'autres études sont nécessaires pour évaluer l'impact clinique en termes d'efficacité de ventilation et de confort.

\section{Références}

1. Javouhey E, Barats A, Richard N, et al (2008) Non-invasive ventilation as primary ventilatory support for infants with severe bronchiolitis. Intensive Care Med 34:1608-14

2. Vignaux L, Grazioli S, Piquilloud L, et al (2013) Optimizing patient-ventilator synchrony during invasive ventilator assist in children and infants remains a difficult task. Pediatr Crit Care Med $14: \mathrm{e} 316-25$ 


\begin{tabular}{|llll|}
\hline Tableau 1 Principaux paramètres et mesures durant 1 min d'analyse & & & \\
& & PAC & \\
\hline Paramètres du respirateur & & & p \\
\hline FiO2 (\%) & $31,5(9,4)$ & $29,2(9,6)$ & 0,22 \\
FR neurale (n/min) & $54,3(19,7)$ & $60,7(19,5)$ & 0,16 \\
FR respirateur (n/min) & $49,8(8,5)$ & $59,5(17,9)$ & 0,03 \\
PEEP (cmH2O) & $5,4(1,1)$ & $5,4(1,2)$ & 0,95 \\
Pression maximale (cmH2O) & $15,2(2,5)$ & $18,7(4,9)$ & 0,09 \\
Eadi max ( $\mu$ V) & $25,3(15,4)$ & $24,1(12,8)$ & 0,80 \\
Auto-déclenchements (n/min) & $8,2(13,3)$ & $0,09(0,3)$ & 0,07 \\
Efforts inefficaces (n/min) & $21,8(16,5)$ & $0,54(1,5)$ & 0,001 \\
Doubles déclenchements (n/min) & $1,0(0,89)$ & $1,56(0,62)$ & 0,28 \\
Index d'asynchronie (\%) & $38(21)$ & $3(3)$ & $<0,0001$ \\
Délai de déclenchement (ms) & $116,0(38,9)$ & $43,9(7,2)$ & $<0,0001$ \\
\end{tabular}

\section{SO022}

\section{Rhabdomyolyse intenses de l'enfant : description clinique et étiologique}

C. Mehler Jacob ${ }^{1}$, M. Oualha ${ }^{2}$, P. de Lonlay ${ }^{3}$, I. Desguerre ${ }^{4}$, P. Hubert ${ }^{2}$, L. Dupic ${ }^{2}$

${ }^{1}$ Neurologie pédiatrique, AP-HP, hôpital de Bicêtre, université Paris-Sud, faculté de médecine Paris-Sud, Le Kremlin Bicêtre, France

${ }^{2}$ Service de réanimation pédiatrique médico-chirurgicale, CHU Necker-Enfants-malades, Paris, France

${ }^{3}$ Centre de référence des maladies métaboliques, CHU Necker-Enfants-Malades, Paris, France

${ }^{4}$ Neuropédiatrie, CHU Necker-Enfants-malades, Paris, France

Introduction : Les rhabdomyolyses sont fréquentes en pédiatrie. Plusieurs publications montrent une augmentation de la fréquence des maladies métaboliques et des complications rénales lorsque le taux de CPK est élevé et une étude établit un seuil à $6000 \mathrm{UI} / \mathrm{L}$. Pourtant, il n'existe aucune description portant sur les enfants qui présentent des CPK particulièrement élevées. L'objectif de notre étude est de décrire la présentation clinique, l'évolution et les étiologies des rhabdomyolyses intenses de l'enfant (CPK supérieures à $6000 \mathrm{UI} / \mathrm{L}$ ) et d'analyser la démarche diagnostique pour en déterminer la cause.

Patients et méthodes : Il s'agit d'une étude rétrospective sur 5 ans portant sur les patients de moins de 18 ans hospitalisés dans un hôpital pédiatrique parisien qui avaient un dosage de CPK supérieur à $1000 \mathrm{UI} / \mathrm{L}$. Les patients ont été identifiés par le système informatique de biochimie de l'hôpital. Les dossiers des patients présentant des CPK supérieures à $6000 \mathrm{UI} / \mathrm{L}$ ont été étudiés.

Résultats : 978 enfants présentant des CPK supérieures à $1000 \mathrm{UI} / \mathrm{L}$ ont été identifiés. Il existait une différence significative de la mortalité : $13 \%$ lorsque les CPK étaient entre 1000 et $6000 \mathrm{UI} / \mathrm{L}$ (849 patients) et $27 \%$ lorsque les CPK étaient supérieures à $6000 \mathrm{UI} / \mathrm{L}$ (129 patients); $\mathrm{p}<0,01$. Il y avait autant d'hospitalisation en réanimation dans les deux groupes. Nous avons étudié 119 dossiers d'enfants qui avaient des CPK à plus de $6000 \mathrm{UI} / \mathrm{L}$. La médiane des CPK maximales était à 13120 UI/L (6 023-690 760). La majorité des patients $(\mathrm{n}=64,54 \%)$ étaient hospitalisés en réanimation, 31 (26\%) en neuro-métabolisme, 8 $(7 \%)$ aux urgences et $16(13 \%)$ dans d'autres services. Ils présentaient une altération de l'état général $(28 \%)$, des myalgies $(21 \%)$ ou des symptômes viraux $(28 \%)$. La moitié des patients $(\mathrm{n}=59)$ étaient intubés ou présentaient des troubles hémodynamiques $(n=59)$. Des troubles de la conscience étaient rapportés chez $15 \%$ d'entre eux $(n=18)$. Au cours de leur séjour, $21 \%(\mathrm{n}=15)$ ont présenté une dysfonction rénale ; $12 \%$ $(\mathrm{n}=13)$ ont été dialysés et $20 \%$ sont décédés. Aucun décès ne pouvait être directement attribué à la rhabdomyolyse.

Pour $50 \%$ des patients $(n=60)$, la cause de la rhabdomyolyse était connue ou évidente à l'arrivée ; pour les autres patients $(\mathrm{n}=59)$ pour lesquels la cause n'était pas évidente, le diagnostic a été déterminé avec certitude dans la moitié des cas $(n=29)$ et est resté incertain chez l'autre moitié $(\mathrm{n}=30)$. Les causes de l'épisode étaient des défaillances viscérales ( $28 \%$ ), des infections $(15 \%)$, des traumatismes $(15 \%)$, une chirurgie lourde (14\%), des maladies métaboliques (10\%), des myopathies $(20 \%)$ et des mutations LPIN1 (3\%). Plusieurs causes associées expliquaient la rhabdomyolyse chez $34 \%$. Pour 14 des 30 patients sans diagnostic certain, aucune exploration métabolique n'avait été réalisée : une infection virale était alors évoquée, sans preuve.

Discussion : Notre étude est la première large description des rhabdomyolyses intenses de l'enfant. Elle confirme qu'il existe une mortalité plus importante lorsque les CPK sont supérieures à $6000 \mathrm{UI} / \mathrm{L}$. La fréquence des maladies métaboliques chez ces patients est plus importante que ce qui est décrit dans les études portant sur toutes les rhabdomyolyses (CPK supérieures à $1000 \mathrm{UI} / \mathrm{L}$ ).

Conclusion : Les patients qui présentent des CPK supérieures à $6000 \mathrm{UI} / \mathrm{L}$ ont une mortalité plus élevée que les patients qui ont des CPK entre 1000 et $6000 \mathrm{UI} / \mathrm{L}$. Ils sont plus susceptibles d'avoir une maladie métabolique que les patients avec des CPK moins élevées. Ces patients doivent donc bénéficier d'explorations métaboliques minimales telles qu'une chromatographie des acides organiques urinaires, un profil des acylcarnitines et un dosage de la carnitine ainsi que d'un avis complémentaire spécialisé.

\section{Bibliographie}

1. Mannix R, Tan ML, Wright R, Baskin M (2006) Acute Pediatric Rhabdomyolysis: Causes and Rates of Renal Failure. Pediatrics 118:2119-25

\section{SO023}

Veno-arterial extra-corporeal membrane oxygenation support for paediatric refractory septic shock

J. Rambaud ${ }^{1}$, I. Guellec ${ }^{2}$, S. Renolleau ${ }^{1}$, P.L. Leger ${ }^{2}$, J. Guilbert ${ }^{2}$

${ }^{1}$ Service de réanimation pédiatrique, CHU Armand-Trousseau,

Paris, France

${ }^{2}$ Réanimation pédiatrique et néonatale, CHU Armand-Trousseau, Paris, France

Introduction: Septic shock is an important cause of mortality in paediatric intensive care units. The American College of Critical 
Care Medicine published guidelines on this topic which have been reinforced in the paediatric section of the first Surviving Campaign guidelines in 2004, as well as in regular updates thereafter. So, in neonates or children with circulatory collapse unresponsive to maximal conventional treatment, ExtraCorporeal Membrane Oxygenation (ECMO) should be considered. The aim of this study was to describe our experience in the management of refractory septic shock requiring ECMO.

Design: Retrospective case series.

Settings: Paediatric intensive care unit in a university children hospital, ECMO referral centre.

Patients and methods: We performed a retrospective study of our ECMO data base to identify patients who received veino-arterial extra-corporeal membrane oxygenation (V-A ECMO) for septic shock from January 2004 to June 2013, in our PICU at Armand-Trousseau Children's Hospital. We included all neonate and children up to 18 years old that received V-A ECMO for circulatory collapse despite fluid resuscitation and inotropic therapy and had clinical signs of infection or isolation of bacterial microorganism. Before cannulation, we recorded the following information: age, gender, site and cause of infection, PELOD score for children and SNAPPE II score for neonates, delay between ICU admission and cannulation, implantation of ECMO under cardiopulmonary resuscitation, pre-ECMO inotrope score, $\mathrm{PaO} 2 / \mathrm{FiO} 2$ ratio, blood gas analyses, blood lactate level, urine output, leukocytes count, activated protein $\mathrm{C}$, procalcitonin, ventilator settings, oxygenation index. We measured time on ECMO, complications on ECMO and survival to hospital discharge. Patients with secondary septic shock who required ECMO primarily for respiratory failure were excluded. Other exclusion criteria for V-A ECMO were a prolonged cardiac arrest ( $>60$ minutes), a severe irreversible neurological pathology such as cranial haemorrhage or flat electroencephalogram tracing before cannulation. All of the statistics cited in this study were produced through a Fisher's exact test. The significance level was set at $\mathrm{p}<0.05$.

Main results: Fourteen neonates were included. In 57\%, shock was due to a Streptococcus B infection and in $36 \%$ to Escherichia coli. One Pseudomonas aeruginosa infection was identified. In one case, infection was not documented. Eight children were included. The medium age was 30 months (1-113 months). Microorganisms identified were: Escherichia coli (1), Streptococcus A (1), Streptococcus pneumonia (1-coinfected with H1N1), Shigella (1), Legionella pneumophilia (1), Enterobacter (1). Fourteen (63\%) of the 22 patients could be weaned from V-A ECMO after an average of 6.6 days (1-17). Half of paediatric patients survived while $64 \%$ of the neonates survived. No correlation was found between death and severity scores (PELOD score, $p=0.94$; SNAPPE II, $\mathrm{p}=0.34)$. Inotrope score before cannulation and ECMO success were not correlated $(p=0.77)$. Blood samples before cannulation showed no correlation between ECMO success and $\mathrm{pH}(\mathrm{p}=0.27)$; lactate level $(\mathrm{p}=0.33), \mathrm{PaO} 2 / \mathrm{FiO} 2$ ratio $(\mathrm{p}=0.49)$, oxygenation index $(p=0.35)$. The average delay of cannulation after arrival in our unit was $11.6 \mathrm{~h}$ and there was no significant difference between survivors and non survivors. No major bleeding was noted during ECMO. Three patients (13.6\%) presented stroke and two $(9 \%)$ evolved to brain death.

Conclusion: Extracorporeal membrane oxygenation can be safely used to resuscitate and support children with refractory septic shock. This study adds support to existing guidelines. An early transfer to an ECMO referral Centre could improve survival.

\section{SO024}

Valeur pronostique précoce des paramètres d'oxygénation dans le sepsis sévère de l'enfant : étude multicentrique française

A. Botte ${ }^{1}$, J. Guichoux ${ }^{2}$, O. Brissaud ${ }^{2}$, S. Teyssedre 3 , J.-M. Liet ${ }^{4}$, S. Leteurtre ${ }^{1}$, F. Leclerc ${ }^{1}$

${ }^{1}$ Service de réanimation pédiatrique, CHRU de Lille,

hôpital Jeanne-de-Flandre, Lille, France

${ }^{2}$ Service de réanimation pédiatrique, CHU Pellegrin Tripode,

Bordeaux, France

${ }^{3}$ Réanimation pédiatrique, hôpital Femme-Mère-Enfant, hospices civils de Lyon, Lyon, France

${ }^{4}$ Service de réanimation pédiatrique, CHU de Nantes, hôpital Mère-Enfant, Nantes, France

Introduction : La mortalité des états septiques de l'enfant reste de 10 à $40 \%$. Rivers chez l'adulte et de Oliveira chez l'enfant [1] ont montré que la surveillance de la saturation en oxygène de l'hémoglobine du sang veineux dans la veine cave supérieure (VCS) $(\mathrm{ScvO} 2)$ associée à une prise en charge précoce réduisait la mortalité respectivement de 46,5 vs $30,5 \%$ et 39,2 vs $11,8 \%$. La valeur pronostique des paramètres microcirculatoires et d'oxygénation ont été peu étudiés à la phase initiale des états septiques graves de l'enfant. L'objectif principal était d'étudier dans les 24ères heures du sepsis grave de l'enfant, la valeur pronostique des paramètres d'oxygénation $(\mathrm{ScvO} 2$, lactatémie et clairance du lactate) en termes de morbimortalité.

Patients et méthodes : Il s'agit d'une étude de cohorte prospective observationnelle multicentrique française réalisée de janvier 2009 à janvier 2012 dans 5 services de réanimation pédiatrique (RP). Étaient inclus les enfants : 1) ayant un sepsis grave défini d'après la conférence de consensus internationale ; 2) pris en charge avant la $12^{\mathrm{e}}$ heure du début de la période d'éligibilité ; 3) dont l'âge était entre 28 jours et 18 ans ; 4) équipés d'un cathéter veineux central situé dans la VCS ; 5) chez lesquels étaient réalisés un dosage du lactate et une mesure de ScvO2. Les critères de jugement étaient la mortalité à J60 de l'inclusion et/ou la présence de séquelles et/ou la décision d'une limitation et arrêt des thérapeutiques (LAT). Le recueil clinique des données diagnostiques et thérapeutiques était réalisé à $\mathrm{H} 0, \mathrm{H} 2, \mathrm{H} 6, \mathrm{H} 12$ et $\mathrm{H} 24$ et la prise en charge reposait sur les recommandations internationales [2]. La clairance du lactate H6 était définie par [lactate H0 - lactate H6]/lactate H0.

Résultats : 47 enfants étaient inclus dont 25 cas de méningocoque $\mathrm{B}$ et 3 de méningocoque $\mathrm{C}$. Les caractéristiques cliniques et les paramètres d'oxygénation sont résumés dans le tableau. Il y avait 7 décès, 6 avant J28. 5 enfants justifiaient une LAT, 21 avaient des séquelles. La clairance du lactate H6 n'était pas liée à la survenue de morbimortalité, contrairement aux clairances du lactate H12 $(p=0,01)$ et H24 $(p=0,01)$.

Conclusion : Dans notre étude, la mortalité et la morbidité étaient respectivement de 15 et $51 \%$. Le principal pathogène était le Méningocoque $B$ $(53 \%)$. Le taux de lactate moyen, les scores inotrope et inotrope-vasoactif moyens, l'IC à H24, le PELOD à H24 et la durée de séjour étaient liés à la survenue du décès à J60, de séquelle ou de LAT, contrairement à la $\mathrm{ScvO} 2$ dans les 24ères heures du sepsis grave de l'enfant.

\section{Références}

1. de Oliveira CF, de Oliveira DS, Gottschald AF, et al (2008) ACCM/ PALS haemodynamic support guidelines for paediatric septic shock: an outcomes comparison with and without monitoring central venous oxygen saturation. Intensive Care Med 34:1065-75

2. Dellinger RP, Levy MM, Carlet JM, et al (2008) Surviving Sepsis Campaign: international guidelines for management of severe sepsis and septic shock: 2008. Crit Care Med 36:296-327 


\begin{tabular}{|c|c|c|c|c|}
\hline Caractéristiques & $\begin{array}{l}\text { Survivants sans séquelle } \\
\text { sans LAT }(n=23) 49 \%\end{array}$ & $\begin{array}{l}\text { Décès J60 } \pm \text { séquelles } \pm \text { LAT } \\
(\mathrm{n}=24) 51 \%\end{array}$ & $\begin{array}{l}\text { Population géné- } \\
\text { rale }(n=47)\end{array}$ & $\mathbf{p}$ \\
\hline Âge (années) & $\begin{array}{l}1,3[0,6 ; 7,6] \\
3,9 \pm 5\end{array}$ & $\begin{array}{l}2,1[0,8 ; 3,7] \\
3,6 \pm 4,5\end{array}$ & $\begin{array}{l}1,5[0,8 ; 3,9] \\
3,8 \pm 4,7\end{array}$ & $\mathrm{p}=0,88$ \\
\hline Poids (kg) & $\begin{array}{l}10[7,5 ; 25] \\
16 \pm 12,7\end{array}$ & $\begin{array}{l}12[8,6 ; 15,1] \\
17,1 \pm 16,1\end{array}$ & $\begin{array}{l}12[7,9 ; 16] \\
16,6 \pm 14,4\end{array}$ & $\mathrm{p}=0,63$ \\
\hline $\operatorname{Sex} \operatorname{ratio}(H / F) n(\%)$ & $13(28) / 10(21)$ & $16(34) / 8(17)$ & $29(62) / 18(38)$ & $\mathrm{p}=0,47$ \\
\hline Index de choc à H24 & $\begin{array}{l}1,3[0,9 ; 1,5] \\
1,2 \pm 0,3\end{array}$ & $\begin{array}{l}1,6[1,2 ; 2,1] \\
1,6 \pm 0,5\end{array}$ & $\begin{array}{l}1,3[1,1 ; 1,7] \\
1,6 \pm 0,5\end{array}$ & $\mathbf{p}=\mathbf{0 , 0 3}$ \\
\hline Clairance du lactate $\mathrm{H} 6$ & $\begin{array}{l}32[-9,4 ; 59,4] \\
7,8 \pm 73,7\end{array}$ & $\begin{array}{l}23[-15,6 ; 53] \\
-22,1 \pm 214\end{array}$ & $\begin{array}{l}26[-13,5 ; 54] \\
-7,8 \pm 162\end{array}$ & $\mathrm{p}=0,52$ \\
\hline $\begin{array}{l}\text { Lactate moyen dans les } 24 \text { pre- } \\
\text { mières heures }(\mathrm{mmol} / \mathrm{l})\end{array}$ & $\begin{array}{l}1,8[1,2 ; 2,6] \\
2,1 \pm 1,2\end{array}$ & $\begin{array}{l}3,1[1,7 ; 4,6] \\
3,5 \pm 1,1\end{array}$ & $\begin{array}{l}2,2[1,3 ; 3,5] \\
3 \pm 2,7\end{array}$ & $p=0,025$ \\
\hline $\begin{array}{l}\mathrm{ScvO} 2 \text { moyenne dans les } 24 \text { pre- } \\
\text { mières heures }(\%)\end{array}$ & $\begin{array}{l}71[68 ; 75] \\
70 \pm 7\end{array}$ & $\begin{array}{l}67[57 ; 74] \\
65 \pm 10\end{array}$ & $\begin{array}{l}69[60 ; 74] \\
68 \pm 9\end{array}$ & $\mathrm{p}=0,7$ \\
\hline $\begin{array}{l}\text { Score inotrope moyen les } 24 \text { pre- } \\
\text { mières heures }\end{array}$ & $\begin{array}{l}4,5[0 ; 10,5] \\
5,6 \pm 6,4\end{array}$ & $\begin{array}{l}13,7[0,4 ; 25] \\
21,1 \pm 25,7\end{array}$ & $\begin{array}{l}6[0 ; 16] \\
17,2 \pm 33,5\end{array}$ & $\mathbf{p}=\mathbf{0 , 0 2}$ \\
\hline $\begin{array}{l}\text { Score inotrope-vasoactif moyen } \\
\text { les } 24 \text { premières heures }\end{array}$ & $\begin{array}{l}26[7 ; 104] \\
61 \pm 69\end{array}$ & $\begin{array}{l}85[38 ; 234] \\
485 \pm 1700\end{array}$ & $\begin{array}{l}72[20 ; 149] \\
282 \pm 1222\end{array}$ & $\mathbf{p}=\mathbf{0 , 0 1}$ \\
\hline Durée de séjour en RP (jours) & $\begin{array}{l}3,6[2,1 ; 6,3] \\
4,8 \pm 3,8\end{array}$ & $\begin{array}{l}10[4,5 ; 20,3] \\
30,4 \pm 78,2\end{array}$ & $\begin{array}{l}5,9[2,6 ; 14,2] \\
17,9 \pm 56,9\end{array}$ & $\mathbf{p}=\mathbf{0 , 0 1}$ \\
\hline PELOD (\%) & $\begin{array}{l}0,2[0,02 ; 1,7] \\
10,8 \pm 26,3\end{array}$ & $\begin{array}{l}1,7[0,2 ; 32,3] \\
16,8 \pm 26,6\end{array}$ & $\begin{array}{l}0,9[0,03 ; 21] \\
13,9 \pm 26,3\end{array}$ & $p=0,05$ \\
\hline
\end{tabular}

